\title{
Relaxation of Kohn-Sham orbitals of organometallic complexes during the approach of a nucleophilic reactant (or an electron approach): The case of [ $[\mathrm{sal}(\mathrm{ph}) \mathrm{en}]_{2} \mathrm{Zn}$ complexes
}

\author{
Walid Lamine, ${ }^{\mathrm{a}, \mathrm{b}}$ Salima Boughdiri, ${ }^{\mathrm{b}}$ Lorraine Christ ${ }^{\mathrm{c}}$, Lynda Merzoud $^{\mathrm{a}}$, Christophe Morell $^{\mathrm{a}^{*}}{ }^{1}$, \\ Henry Chermette $\mathrm{a}^{*}{ }^{2}$
}

${ }^{a}$ Université de Lyon, Institut des Sciences Analytiques, UMR CNRS 5280, Université Claude Bernard Lyon 1, 69622 Villeurbanne Cedex, France

${ }^{b}$ Université de Tunis El Manar, Faculté des Sciences de Tunis, UR11ES19 Unité de recherche Physico-Chimie des Matériaux Condensés, El-Manar II, 2092, Tunis, Tunisie

c Université de Lyon, Institut de Recherches sur la Catalyse et l'Environnement de Lyon, IRCELYON, UMR CNRS 5256, Université Lyon 1, 69626 Villeurbanne Cedex, France

\begin{abstract}
:
In a recent paper, the Lewis acidic character of a series of $\mathrm{Zn}-\mathrm{Sal}(\mathrm{ph})$ en complexes was reviewed, using conceptual density functional theory descriptors to assess the acidic character. It was shown that the nature of the bridging diamine link in the Schiff base ligand controls this character that is mainly responsible for the coordination of the $\mathrm{Zn}^{2+}$, hence for the geometry of these complexes. However, the usual dual descriptor did exhibit significant weaknesses to retrieve the electrophilic part on the metal cation of the $\mathrm{Zn}$-sal(ph)en complexes. Indeed, it is necessary to include the densities of the electronic excited states through the so-called state specific dual descriptor. This procedure will allow us to recover successfully the appropriate reactivity of the studied complexes holding diamine bridges differing by flexible to semi-rigid and to rigid ranges. Nevertheless, the selection of the excited state allowing a meaningful description of the Lewis acidic is not a priori obtained from a direct identification of the Kohn-Sham (KS) orbitals involved in the excitation. The present work reports an analysis of the relaxation of the KS orbitals when a fraction of charge is added to a virtual orbital, and when an excitation is considered while a fractional charge is transferred from an occupied orbital towards a virtual orbital.
\end{abstract}

\section{Keywords}

$\mathrm{Zn}^{2+}$; salen; salphen; complex; DFT; conceptual DFT; electronic relaxation

\section{Introduction:}

In a recent paper [1] the coordination geometry of $\mathrm{Zn}^{2+}$ cation complexed by Sal(ph)en ligands was analyzed using Conceptual DFT descriptors. Whereas the structure is mainly governed by a tetrahedral geometry which agrees well with the 18 electrons rule, square pyramidal geometries may be found. Indeed, the divalent zinc ion with its filled up $\mathrm{d}^{10}$ configuration is not sensitive to ligand field stabilization effects [2] and consequently its coordination geometry can be somewhat flexible, driven by the nature of charges and ligands.

\footnotetext{
${ }^{1}$ Christophe.Morell@univ-lyon1.fr

2 Henry.Chermette@univ-lyon1.fr
} 
In a first approach, the prototype $\mathrm{ZnCl}_{4}{ }^{2-}$ complex was studied and it was shown that the dual descriptor (DD), first introduced by Morell [3-6], was fully qualified to describe the Lewis acidic behavior of this complex, through a strong electrophile domain localized on the $\mathrm{Zn}$ atom [1]. However, with tetradentate $\mathrm{N}_{2} \mathrm{O}_{2}$ or $\mathrm{N}_{4}$ sal(ph)en-type ligands, in case of a rigid ligand framework, the steric properties of the ligand backbone can force the $\mathrm{Zn}$ divalent cation into an unfavorable square-planar environment, leading to a high Lewis acidity on the metal center [4]. Therefore, in that cases, the $\mathrm{Zn}-\mathrm{Sal}(\mathrm{ph})$ en complexes exhibit a preference to a penta-coordination geometry which would lead to a 20 electrons environment with an axial $\mathrm{N}$ or O-donor ligand added to a quasi-square planar geometry. In case of absence of an axial ligand, the coordinative saturation is then achieved through reciprocal intermolecular $\mathrm{Zn}--\mathrm{O}$ interactions between monomeric $\mathrm{Zn}$-sal(ph)en complexes leading to a dimeric structure with a typical $\mathrm{Zn}_{2} \mathrm{O}_{2}$ central unit [5-8]. Accordingly, the Lewis acidic character of $\mathrm{Zn}^{2+}$ ion is boosted by the rigid coordination geometry of the salphen ligand.

On the other hand, with a sufficient flexible Schiff base ligand, a tetrahedral coordination geometry is retained, leading to helical structures [12-16, 17] via self-assembly processes.

In this framework, the Lewis acidic character of bis(salicylaldiminato)zinc (II) Schiff base complexes has been investigated recently by Forte et al. [15]. The latter have shown how the Lewis acidic character dictates the observed aggregation/disaggregation features of these zinc (II) complexes, which is essential for understanding their catalytic and sensing properties. Even more recently, some of us attempted to understand the Lewis acidic behavior. Indeed, the study of the main factor of the reactivity and of the coordination of $\mathrm{Zn}$-sal(ph)en complexes, using conceptual density functional theory (DFT) local descriptors, is able to tackle regio and stereo selectivity.

The paper is organized as follows: After recalling the theoretical grounds of the Conceptual DFT descriptors discussed in this work, the description of the reactivity of Zn-salen complexes will be summarized. In the following part, the differences in the description of the usual and state specific DD will be presented, and in a third part, the relaxation of the orbitals occurring in the calculation of the DD in its linearized approximation will be discussed. In the next part, the use of fractional occupation will be introduced and assessed. Finally, in a fifth part, we will show that the ligand-metal charge transfer (LMCT) excitation energies are recovered from an analysis of the metal character among the virtual orbitals. A short conclusion will open perspectives.

\section{THEORETICAL BACKGROUND}

Conceptual DFT has been reviewed in several papers $[19,20]$, so we only recall the definitions of the local descriptors which are relevant to the present work, i.e. the Fukui functions and its derivative with respect to the number of electrons, namely the dual descriptor [3].

As is now well known, the Fukui function is defined as the first derivative of the electron density with respect to the number of electrons. It is also connected to the response of the electronic chemical potential $\mu$ with respect to the variation of the external potential at a specific point within the molecule, through a Maxwell relation

$$
f(\boldsymbol{r})=\left(\frac{\partial \rho(\boldsymbol{r})}{\partial N}\right)_{\nu(\boldsymbol{r})}=\left(\frac{\partial \mu}{\partial \nu(\boldsymbol{r})}\right)_{N}
$$


Owing to the derivative discontinuity [18] of the electronic density with respect to the number of electrons, Yang and Parr [19,20] proposed left and right derivatives. The right derivative, when the species undergoes a decrease of the number of electrons, is used to model an attack by a nucleophile. The left derivative characterizes an electrophilic attack. Using the approximation of the derivative by a finite difference of densities of one electron, one gets

Nucleophilic attack

$$
f^{+}(\boldsymbol{r})=\left(\frac{\partial \rho(\boldsymbol{r})}{\partial N}\right)_{\nu(\boldsymbol{r})}^{+} \rightarrow f^{+}(\boldsymbol{r}) \approx \rho_{N+1}(\boldsymbol{r})-\rho_{N}(\boldsymbol{r})
$$

Electrophilic attack

$$
f^{-}(\boldsymbol{r})=\left(\frac{\partial \rho(\boldsymbol{r})}{\partial N}\right)_{\nu(\boldsymbol{r})}^{-} \rightarrow f^{-}(\boldsymbol{r}) \approx \rho_{N}(\boldsymbol{r})-\rho_{N-1}(\boldsymbol{r})
$$

Therefore, the DFT approach to chemical reactivity theory indicates that the reactive site of an electrophile is related to a large positive value of the Fukui function $f^{+}(\boldsymbol{r})$, when, simultaneously, nucleophilic sites are related to $f^{-}(\boldsymbol{r})$. Later, Morell et al. [3] have proposed the so-called dual descriptor, which lets obtain simultaneously nucleophilic and electrophilic local sites and thus correctly predict the site reactivity in molecular systems. Starting from its definition as the second derivative of the electron density function with respect to the number of electrons,

$$
f^{2}(\boldsymbol{r})=\left(\frac{\partial^{2} \rho(\boldsymbol{r})}{\partial N^{2}}\right)_{v(\boldsymbol{r})}=\left(\frac{\partial f(\boldsymbol{r})}{\partial N}\right)_{v(\boldsymbol{r})}
$$

several approximations can be derived from the arithmetic difference between the nucleophilic and electrophilic Fukui functions.

$$
f^{(2)}(\boldsymbol{r})=f^{+}(\boldsymbol{r})-f^{-}(\boldsymbol{r})
$$

Large positive (negative) values of the dual descriptor are found for atomic sites within a molecule that are more electrophilic (nucleophilic) than nucleophilic (electrophilic).

The first level of approximation of this descriptor involves finite differences of densities of systems containing molecular systems into the total number of electrons is increased or decreased by one electron, i.e. by combination of equations $(2 a)$ and $(2 b)$

$$
f^{(2)}(\boldsymbol{r}) \approx \rho_{N+1}(\boldsymbol{r})+\rho_{N-1}(\boldsymbol{r})-2 \rho_{N}(\boldsymbol{r})
$$

Such a level of approximation involves a strong relaxation of the orbitals, particularly in case of organometallic compounds, and more generally systems containing transition metals. To prevent that, a second level of 
approximation is often used, consisting in keeping frozen all orbitals from the (usually neutral) system. In that case, the DD is just the difference of density between the frontier orbitals:

$$
f_{i}^{(2)}(\boldsymbol{r}) \approx \varphi_{L U M O}^{2}-\varphi_{H O M O}^{2}
$$

Numerous examples have shown that this approximation of the dual descriptor works perfectly well for closed shell systems (usually organic systems) whereas it fails to predict the right chemistry of open shell systems such as transition metal complexes. Recently, to overcome this issue, state specific dual descriptors have been introduced [21]. They are grounded upon the difference between excited state electron densities and the ground state density [21]. The state specific dual descriptor (SSDD) of state (i) is computed through:

$$
f_{i}^{(2)}(\boldsymbol{r})=\rho_{i}(\boldsymbol{r})-\rho_{0}(\boldsymbol{r})
$$

in which $\rho_{i}(\boldsymbol{r})$ and $\rho_{0}(\boldsymbol{r})$ stand respectively for the density of the excited state $\mathrm{i}$ and the density of the ground state. Indeed, here again, the frozen orbital approximation is often used, and the working equation becomes:

$$
f_{i}^{(2)}(\boldsymbol{r}) \approx \varphi_{L U M O+x}^{2}(\boldsymbol{r})-\varphi_{\text {HOMO-y }}^{2}(\boldsymbol{r})
$$

where $\varphi_{L U M O+x}^{2}(\boldsymbol{r})$ and $\varphi_{H O M O-y}^{2}(\boldsymbol{r})$ stand respectively for the density of the main two orbitals involved in the excitation, namely the LUMO $+x, x=0,1,2,3 \ldots$ and the HOMO-y, $y=0,1,2,3, \ldots$ In case of $x=y=0$, the SSDD is just the standard DD.

Thanks to such descriptors, the trans effect in octahedral complexes [22] has been revisited efficiently.

\section{Computational details:}

Density functional theory calculations were carried out using the Amsterdam Density Functional (ADF) program developed by Baerends and coworkers [23,24]. All the parameters (functional, basis set, numerical thresholds, etc) were those used in ref. [1] Electron densities needed to compute either the usual DD or the SSDD were obtained by performing single point calculations at optimized geometries in their ground state. Medium grid option has been specified as defaults parameter to all computed cubes files, in order to get both good accuracy and reasonable computational time. In all pictures, for both usual DD and SSDDs, the electrophilic zones are represented in blue, displaying a positive value of the function $f^{(2)}(\boldsymbol{r})>0$ whereas the nucleophilic areas with $f^{(2)}(\boldsymbol{r})<0$ are in light brown. TD-DFT calculation of electronic spectrum of Compound (A) were performed with similar parameters.

\section{Results and Discussion}


We will report in the following figures of five $\mathrm{Zn}^{2+}$ complexes, four of them being with sal(ph)en ligands, together with $\left[\mathrm{ZnCl}_{4}\right]^{2-}$ as a prototype model. In case of salen ligand, the diamine link between the two Schiff bases is flexible (ethyl, cyclohexyl) whereas, in case of salphen ligand, it is stiff (phenyl, pyridine), as described in Scheme 1.

\section{$\left[\mathrm{ZnCl}_{4}\right]^{2-}$ prototype model compound}

As already shown in ref [1] $\left[\mathrm{ZnCl}_{4}\right]^{2-}$ is used as a simple prototype in order to highlight the Lewis acid character of $\mathrm{Zn}$ metal center. Molecular Orbitals (MOs) diagram shows that the Lewis acid character involves the LUMO which is essentially located on the $\mathrm{Zn}$ center ( $\mathrm{Zn} 4 \mathrm{~s}$ character). DD picture (Fig. 1) illustrates the point unambiguously. Use of alternate approximations for the DD does not significantly modify the picture.

The difference with the result obtained with use of eq. (6) comes from the fact that the HOMO is degenerate ( $\mathrm{t}_{2}$ irrep in Td group), so that only one component was retained to describe the HOMO.

\section{Zn-Sal(ph)en complexes}

Here, the differences in the four complex structures are related to the axial zone above the $\mathrm{Zn}$ cation located on more or less distorted square planar skeletons. The electronic demand for the axial binding to the $\mathrm{Zn}^{2+}$ ion in these complexes is the origin of the Lewis acidic character induced by the specific shape of the diamine bridge.

One see clearly in Figs. 2 that the usual DD does not describe satisfactorily this feature, and that the two levels of approximations (eq. (5) and (6)) do not lead to comparable descriptions.

Two causes can be put forward and will be analyzed successfully:

i. The LUMO does not involve anymore the $\mathrm{Zn} 4 \mathrm{~s}$ orbital, responsible of the Lewis acidic character of the complex. In Fig. 3 are reported the MO diagram of complexes $\mathbf{A}$ and $\mathbf{C}$ where it appears that the $\mathrm{Zn} 4 \mathrm{~s}$ character dominates virtual orbitals $\mathrm{LUMO}+4$ or LUMO+6, respectively, while the LUMOs of the $\mathrm{Zn}$-Sal(ph)en complexes are mainly dominated by a ligand character. This explains why the usual DD in its two approximations (eq. 6,8) fails to provide the expected chemical reactivity of such complexes.

ii. In case of the calculation of the DD through eq. (5), a strong relaxation occurs among the orbitals when one electron is removed from the HOMO (or below), and/or added to the LUMO (or above). E.g. swaps between virtual orbitals lead to (semi-occupied new) LUMO exhibiting a different character, a different electron localization with respect to what was in the (usually neutral) ground state of the molecular system. It has been shown in ref. [21] that the use of the SSDD lets recover the expected Lewis acid character, provided that the convenient excited state is retained.

Therefore, the SSDD allows overcoming these limitations by involving molecular orbitals other than the frontier ones. For instance, in the system represented in Fig. 3, one has to select excitations towards LUMO+4 or LUMO+6, respectively. As just said, for small systems, the SSDD obtained by eq. (5) and eq. (6) are qualitatively the same, 
whereas, on the contrary, they are different in larger systems because, during the excitation, the orbitals are shuffled through the relaxation process, leading to, if no care is taken, obtained excited densities not describing the expected excitation.

Accordingly, when the excited states are carefully selected, one can see in Fig. 4 (SSDD with frozen orbital approximation) that significant Lewis acidity differences between $\mathrm{Zn}$-sal(ph)en complexes can be observed related to the rigidity of the sal(ph)en ligand:

Complexes $\mathbf{A}$ and $\mathbf{B}$ exhibit a low electrophilicity on the $\mathrm{Zn}$ metal center, whereas complexes $\mathbf{C}$ and $\mathbf{D}$ exhibit a high electrophilicity on the $\mathrm{Zn}$ metal center, and therefore a propensity to axial coordination. These features were the object of discussions in ref. [1]. Let us recall that, for the purpose, the SSDD approximation used was eq. (8), namely the frozen orbital approximation, consisting in the difference of densities of the sole two orbitals involved in the electronic transition HOMO-y $\rightarrow$ LUMO $+x$ (with $y=0$ in that case). This approximation provides a description of the early step of the excitation. On the contrary, the linearized approximation (eq. 7) includes some electronic relaxation, which may be strong, through the calculation of the relaxed density of the cation and the anion.

It is then tempting to force the MOs possessing a given character (the $\mathrm{Zn} 4 \mathrm{~s}$ in our case) to keep their occupation during the electronic relaxation process. Within ADF, an algorithm was implemented in the eighties (the "Keeporbitals" keyword) for the purpose. Such algorithms which let the orbital occupations follow the orbital character (i.e. the MO coefficients) during the SCF process have early been developed in the eighties to calculate semi-core ionization energies [25] in MS-LSD code [26] and other codes. With ADF, the "Keeporbitals keyword" is switched on automatically if fractional numbers are used. A similar algorithm ("Maximum Overlap Method") has also been published more recently for codes handling GTOs. [27] However, care should be taken, as some convergence accelerators may overcome this feature, leading to wrong excited states. Recently it has been suggested [28] to consider the maximisation of the orbital overlaps between iteration $n$ and iteration 1 during the SCF process, instead of between iteration $\mathrm{n}$ and iteration $\mathrm{n}-1$. We have not (yet) implemented such algorithm, which, however, may not lead to different results.

However, this is less straightforward than anticipated, because some convergence accelerators may overcome this feature, not leading to the expected excited states. Indeed, a major side effect of the relaxation (mainly for the anion) is to redistribute the $\mathrm{MO}$ characters, with a spillover of the (in our case, the $\mathrm{Zn} 4 \mathrm{~s}$ ) character among several MOs. Such a feature indicates that the excited state is less correctly described by a single Kohn-Sham determinant than the ground state is, and this is interestingly similar to what is obtained in TD-DFT calculations of optical spectra. This point will be addressed in the last part of this paper.

A crucial point in the calculation of SSDDs is the selection of the excited state the most pertinent to describe the reactivity. Since the relaxation of the MOs is large when one electron is added to, or removed from, the molecular systems, one be tempted to use an approximation closer to its definition (eq. 3), namely the derivative of the density with respect to the number of electrons. For the purpose, one could investigate the relaxation process through the addition or subtraction of a small amount of electron, by example $0.05 \mathrm{e}$, an amount sufficient to induce significant relaxations, without shuffling too strongly the MO energy panel and the MO composition. The use of fractional occupation is easy to handle with DFT codes, provided one stays at LDA or GGA levels, (i.e. not with 
hybrid or double hybrid functionals). As already mentioned, in eq. (5) the dual descriptor DD is approximated by the finite differences of densities of systems containing molecular systems with a total number of electrons increased or decreased by one electron. This is the finite differences approximation of a second derivative. However, the SSDD approximation introduced in eq. (7) [ref 24] is the difference between the excited state and the ground state, i.e the densities of the depleted orbital (HOMO or below) and the populated orbital (LUMO or above). This SSDD approximation is based on the frozen orbitals approximation. The SSDD approximation used in eq. (10) is the same approximation of second derivative of the energy with respect to the number of electron, but using a much smaller difference in the electron numbers. Here, $0.05 \mathrm{e}$ is an amount sufficient to induce significant relaxations, without shuffling too strongly the MO energy panel and the MO composition

Accordingly, the DD would be approximated by:

$$
f^{(2)}(\boldsymbol{r}) \approx \rho_{N+0.05}(\boldsymbol{r})+\rho_{N-0.05}(\boldsymbol{r})-2 \rho_{N}(\boldsymbol{r})
$$

The SSDD of state (i) is then computed through:

$$
f_{i}^{(2)}(\boldsymbol{r})=\rho_{i}^{+0.05}(\boldsymbol{r})+\rho_{0}^{-0.05}(\boldsymbol{r})-2 \rho(\boldsymbol{r})
$$

in which $\rho_{i}^{+0.05}(\boldsymbol{r})$ and $\rho_{0}^{-0.05}(\boldsymbol{r})$ stand respectively for the density of the excited state i populated by 0.05 electron, and the density of the ground state depopulated by 0.05 electron. In case of the $\left[\mathrm{ZnCl}_{4}\right]^{2-}$ prototype, one can see the differences in Fig. 1 in which the fractional occupation leads to qualitatively similar lobes as those using eq. (5), as expected.

For complexes bigger than $\left[\mathrm{ZnCl}_{4}\right]^{2-}$, e.g. sal(ph)en complexes, the most noticeable result is that the reactivity is not anymore controlled by the DD governed by the HOMO and LUMO, but by MOs that have relaxed, and kept their electrophile/nucleophile character, i.e. those that have to be selected for the state specific dual descriptor (SSDD). The relaxation observed when eq. (7) is used appears already when a small amount of electron (0.05 e-) is involved.

This use of fractional occupation immediately leads to moderate relaxation of the density. However, one can already observe also that some MOs relax more than others do. In Fig. 5, the orbital relaxation energies are reported for MOs of the A (Ethyl linked) complex whose electron numbers have been altered by $0.05 \mathrm{e}$ - either by addition to the LUMO, or by subtraction from the HOMO. It is easy to observe that all orbitals relax, but some relax more even if the occupation change does not concern these orbitals.

A global trend of smaller relaxation for the highest (empty) levels i observed. This result is expected, since the highest virtual orbitals, possessing high energy, are more and more delocalized. 
The analysis of the MO relaxation of compound A during an excitation shows that, in case of a HOMO $\rightarrow$ LUMO excitation by $0.05 \mathrm{e}-$, the most relaxed MO is \#91 i.e. LUMO+6 (and LUMO+8). This observation is not in agreement with the most efficient SSDD involving the LUMO+4 (see Fig. 3 , lhs), and which should be used when approximate eq. (8) is used.

Similar results are obtained for other complexes, as reported in Fig. 7-9

In order to analyze the MOs shuffling occuring for the A molecule, we report in Table 1 the changes in the $\mathrm{Zn} 4 \mathrm{~s}$ character occurring of the virtual orbitals of salen compound $\mathbf{A}$ when a single electron is added to the LUMO, LUMO +1, LUMO $+2 \ldots$ orbitals. The obtained results show that the character of the virtual orbitals changes significantly. In the same time, the addition of an electron to the MO having a character dominated by $\mathrm{Zn}$, will give it a character that is spilled over orbitals below and above it.

Table 1. Evolution of the Zn 4 s character of the virtual MOs with the occupation by a single electron of one virtual MO of the ethyl complex (A). HOMO is \#84. E.g. Column 89 gives the Zn 4 s character of 84-95 MOs through addition of one electron in $M O \# 89$

\begin{tabular}{c|cccccccccc} 
Occ MO \# & $\mathbf{8 4}$ & $\mathbf{8 6}$ & $\mathbf{8 7}$ & $\mathbf{8 8}$ & $\mathbf{8 9}$ & $\mathbf{9 0}$ & $\mathbf{9 1}$ & $\mathbf{9 2}$ & $\mathbf{9 3}$ \\
\hline MO \# & 0 & 0 & 0 & 0 & 0 & 0 & 0 & 0 & 0 \\
$\mathbf{8 4}$ & 0 & 0 & 0 & 0 & 0 & 0 & 0 & 0 & 0 \\
$\mathbf{8 5}$ & 0 & 0 & 0 & 0 & 0 & 0 & 0 & 0 & 0 \\
$\mathbf{8 6}$ & 0 & 0 & 0 & 0 & 0 & 0 & 0 & 0 & 0 \\
$\mathbf{8 7}$ & 0 & 0 & 0 & 0 & 0 & 0 & 0 & 0 & 0 \\
$\mathbf{8 8}$ & 0 & 0 & 0 & 0 & 16.5 & 0 & 0 & 0 & 0 \\
$\mathbf{8 9}$ & 0 & 3.8 & 20.9 & 36 & 2.7 & 24 & 0 & 15.2 & 14 \\
$\mathbf{9 0}$ & 0 & 3.5 & 5.5 & 0 & 6.37 & 22.1 & 59 & 32.9 & 25.1 \\
$\mathbf{9 1}$ & 0 & 10.8 & 0 & 0 & 14.2 & 8 & 0 & 8.6 & 19.3 \\
$\mathbf{9 2}$ & 0 & 21.5 & 62 & 52 & 36 & 21.3 & 38.6 & 9.1 & 25 \\
$\mathbf{9 3}$ & 0 & 49 & 0 & 0 & 12.6 & 20.1 & 6 & 10.3 & 10.4 \\
$\mathbf{9 4}$ & 0 & 0 & 0 & 0 & 5.3 & 0 & 0 & 19.1 & 0 \\
$\mathbf{9 5}$ & 0 & 0 & 0 & 0 & 7.3 & 7.7 & 0 & 5.5 & 10.9 \\
\hline
\end{tabular}

Considering that, the ligand-metal charge transfer transitions belong to the most intense electronic transitions in such complexes. It would be interesting to see if there is an influence of the data reported in Table 1 and the estimation of the transition energies. The excitation energy from the HOMO towards virtual MOs is calculated as the sum of the difference in the eigenvalues (LUMO+y - HOMO) weighted by the Zn4s character $\omega_{i}$ of the corresponding LUMOs

$$
E=\sum_{i} \omega_{i} \varepsilon_{i}-\varepsilon_{\text {номо }}
$$


(see Table 2). Interestingly, the excitations energies obtained through this procedure are almost the same, whatever being the occupied LUMO, as can be seen in Table 2. Even more interestingly, these excitation energies are similar to what can be provided by a TD-DFT calculation (see Table 2). The analysis of this point will the subject of a further work.

Table 2. Transition energy calculated as the sum of HOMO $\rightarrow$ LUMO+x energies, weighted by the Zn4s character of the LUMO+y orbitals, $y=1, \ldots 12$, and calculated from 1e-occupied LUMO+x orbitals

\begin{tabular}{cc} 
Occupied LUMO $_{+\mathbf{x}}$ & $\mathbf{e V}$ \\
\hline LUMO+2 & 4.31 \\
$\mathrm{LUMO}+3$ & 4.24 \\
$\mathrm{LUMO}+4$ & 4.27 \\
$\mathrm{LUMO}+5$ & 4.16 \\
$\mathrm{LUMO}+6$ & 4.38 \\
LUMO+7 & 4.32 \\
LUMO+8 & 4.32 \\
\hline average & 4.29 \\
sigma & 0.071 \\
\hline
\end{tabular}

TDDFT excitations with significant oscillator strength:

$\begin{array}{ll}\text { Excitation \# } 13 & 4.245 \\ \text { Excitation \# } 14 & 4.375\end{array}$

\section{Concluding remarks:}

In this article, the relaxation process occurring through an excitation described by the transfer of a single electron between two Kohn-Sham orbitals has been investigated. This is important for the behavior of chemical reactivity descriptors defined in the so-called Conceptual DFT. For the purpose the modelling of the Lewis acidic character of $\mathrm{Zn}$ metal center in $\mathrm{Zn}$-sal(ph)en complexes has been analyzed. Especially, both usual dual descriptor and state specific dual descriptor have been applied to the description of the electrophilic character of $\mathrm{Zn}$-sal(ph)en complexes. It was shown that the state specific dual descriptor, through the inclusion of densities of the excited states, is necessary to get a satisfactory description of the electrophilic/nucleophilic distributions among the complexes. However, for small complex like $\mathrm{ZnCl}_{4}{ }^{2-}$, the usual dual descriptor provides already a significant description. The relaxation of all the (occupied, virtual) molecular orbitals has been evidenced. Thus, it has been shown that the choice in the approximation retained for the calculation of the dual (densities differing by integer number of electrons, by small fractional amount of electrons, or frozen orbitals) may be crucial to obtain significant and satisfactory description of the chemical reactivity. 
Scheme 1. Structures of Zn-sal(ph)en complexes with various diamine bridges:

Ethyl(A), Cyclohexyl (B), Phenyl(C), anilyl (D).
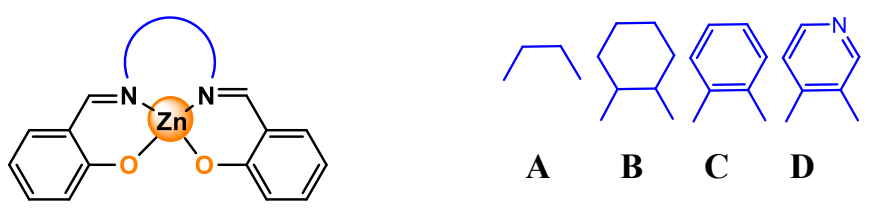

A $B$ C D 

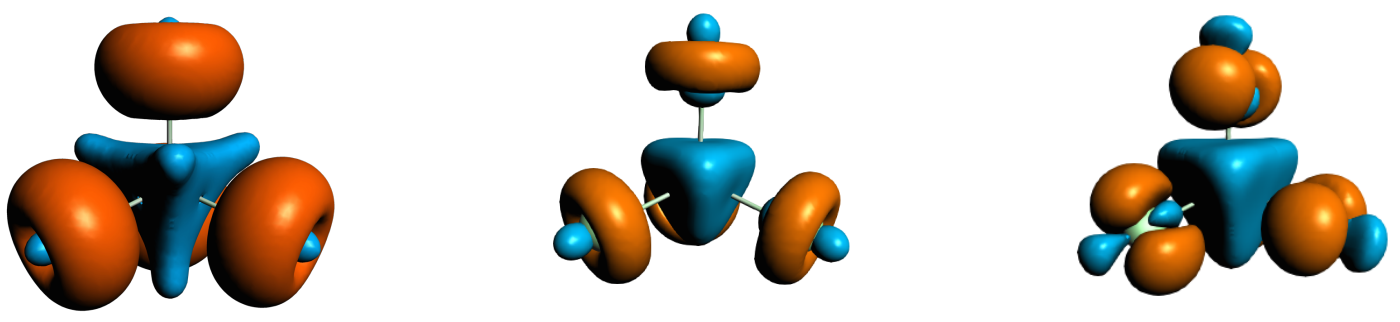

Fig. 1 Usual dual descriptor for [ $\left.\mathrm{ZnCl}_{4}\right]^{2-}$ prototype (T $T_{d}$ geometry): DD approximated according to: (left) eq. (5) $f^{(2)}(\boldsymbol{r}) \approx \rho_{N+1}(\boldsymbol{r})+\rho_{N-1}(\boldsymbol{r})-2 \rho_{N}(\boldsymbol{r})$, (middle) eq. (9) $f^{(2)}(\boldsymbol{r}) \approx \rho_{N+0.05}(\boldsymbol{r})+\rho_{N-0.05}(\boldsymbol{r})-$ $2 \rho_{N}(\boldsymbol{r})$ and (right) $e q(6) f_{i}^{(2)}(\boldsymbol{r}) \approx \varphi_{\text {LUMO }}^{2}-\varphi_{\text {HOMO }}^{2}$, (from left to right) (isovalue $=0.01$ a.u.). 

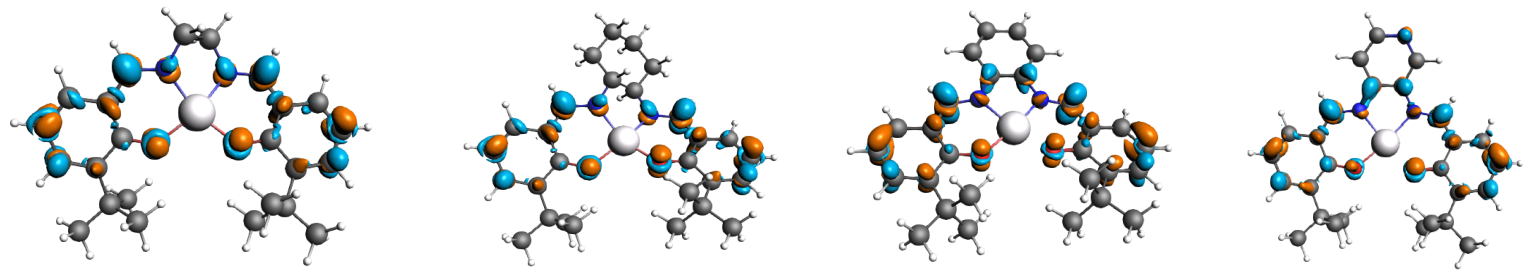

Fig. 2a DD of Zn selected sal(ph) complexes calculated according eq. (5); isovalue 3.10-3 a.u.
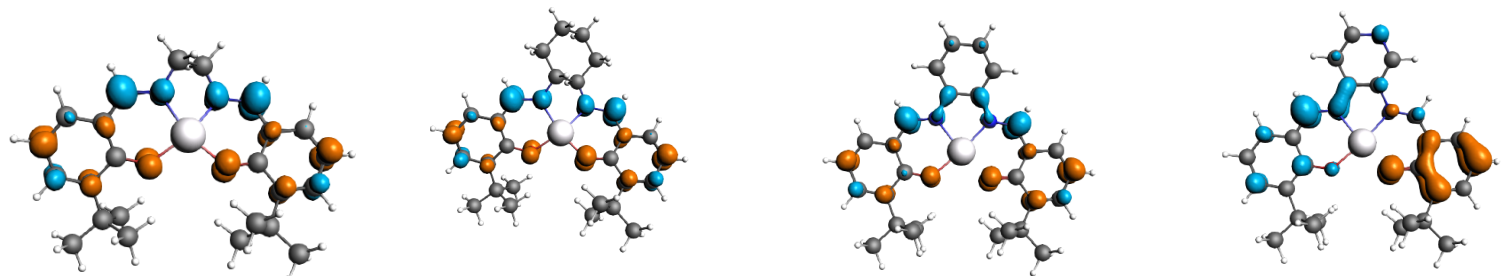

Fig. $2 \boldsymbol{b}$ DD of Zn selected sal(ph) complexes calculated according eq. (6); isovalue 3.10 $0^{-3}$ a.u. 
A

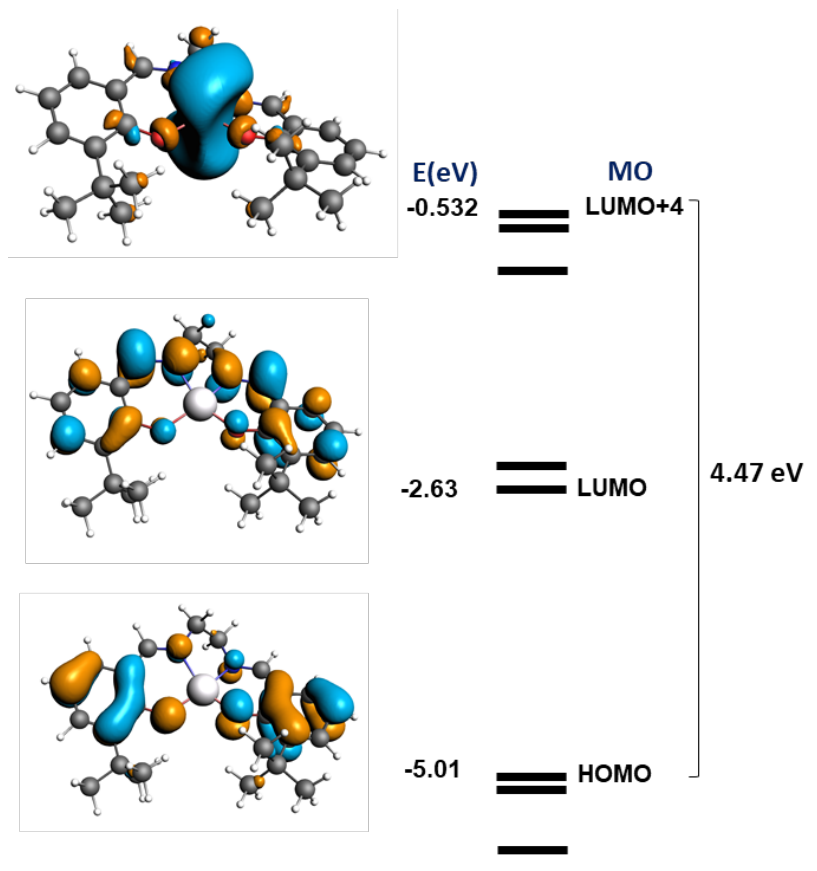

C

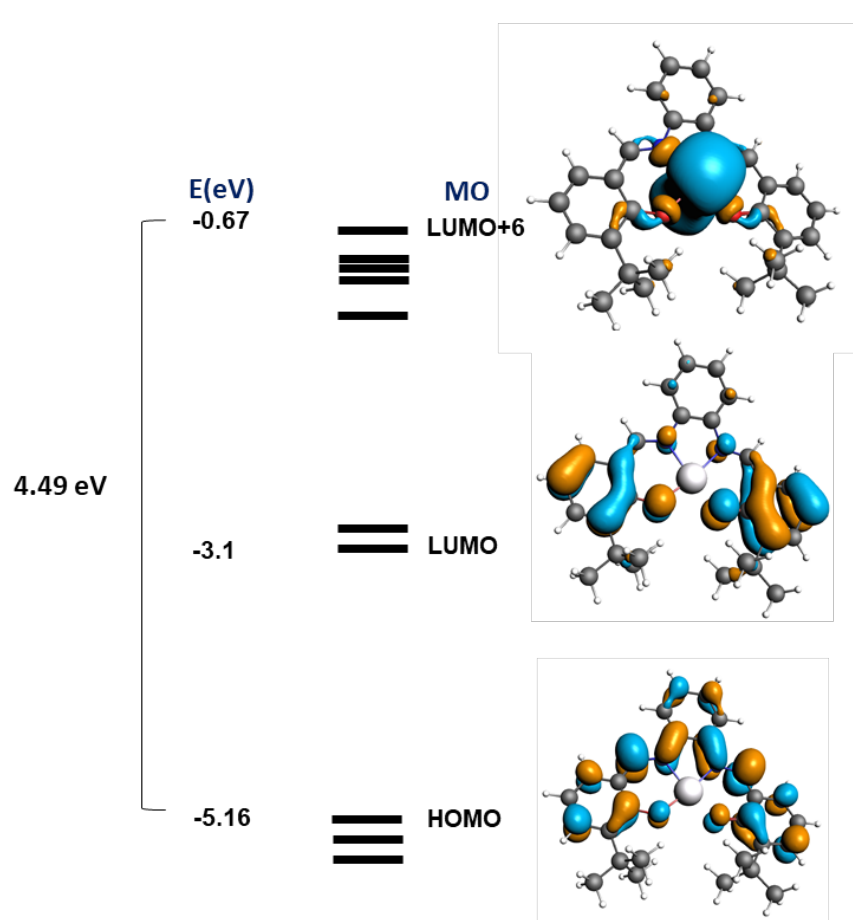

Fig. 3: Frontier MOs and energies (eV) for optimized structures of two chosen Zn-sal(ph)en complexes with ethylenediamine (A) and phenylenediamine (C) as diamine bridge. 


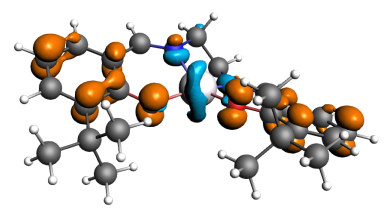

A

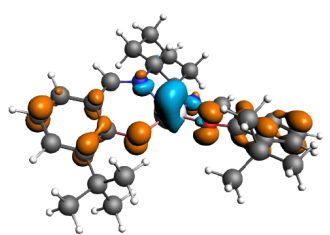

B

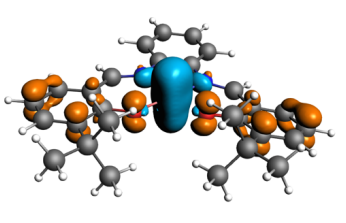

C

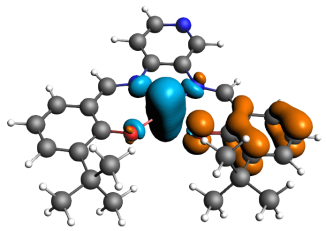

D

Fig. 4 SSDD for the $n^{\text {th }}$ excited state of the investigated Zn-Sal(ph)en complexes with various diamine bridge (Ethyl(A), Cyclohexyl(B), Phenyl(C), Pyridyl(D)) (isovalue $=3.10^{-3}$ a.u.) 


\section{LUMO + 0.05e}

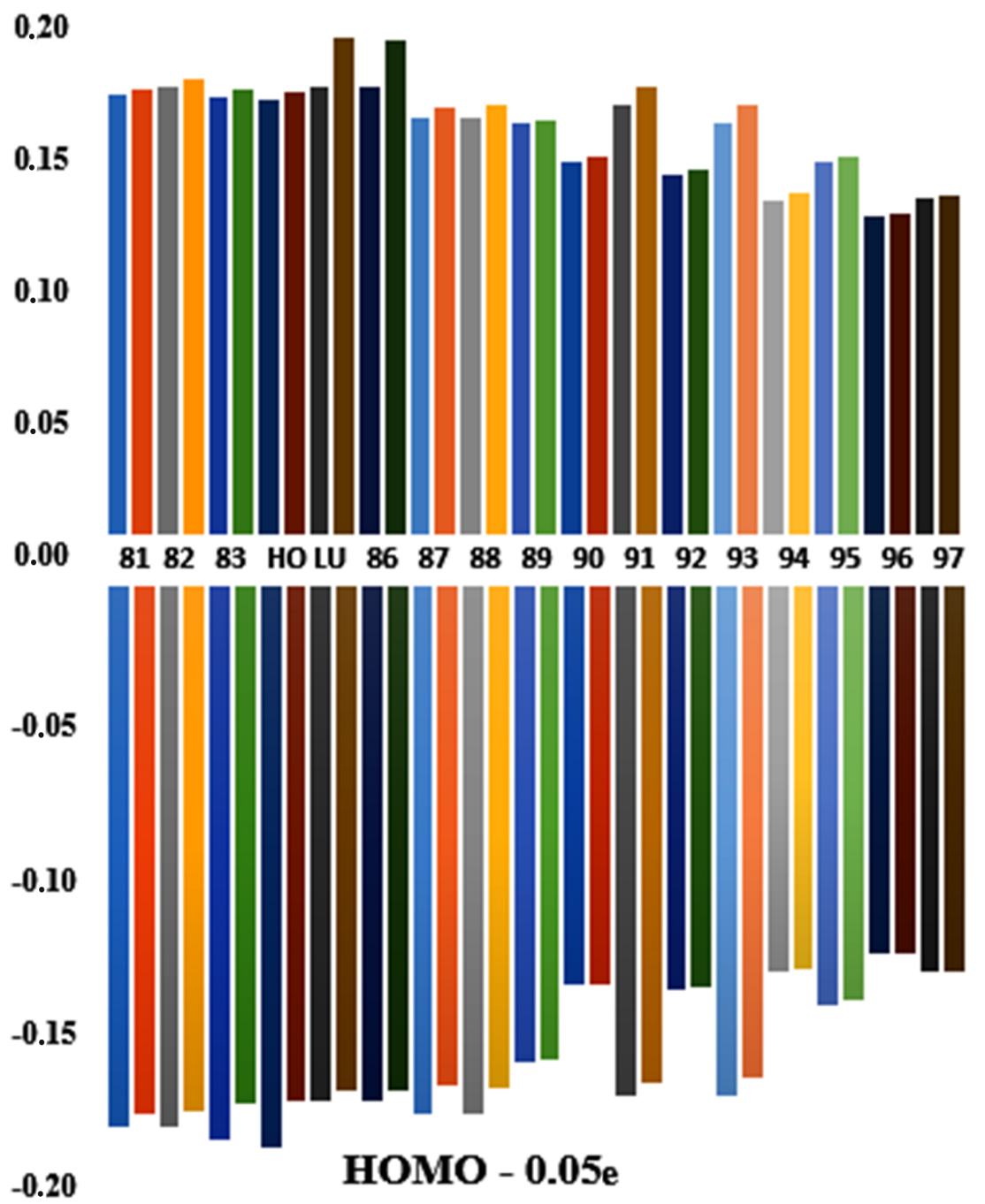

Fig. 5 Relaxation of the $(\alpha, \beta)$ orbitals of the $\boldsymbol{A}$ complex when frontier orbitals are altered by 0.05 electron occupancy. (Eigen value shifts in eV). 


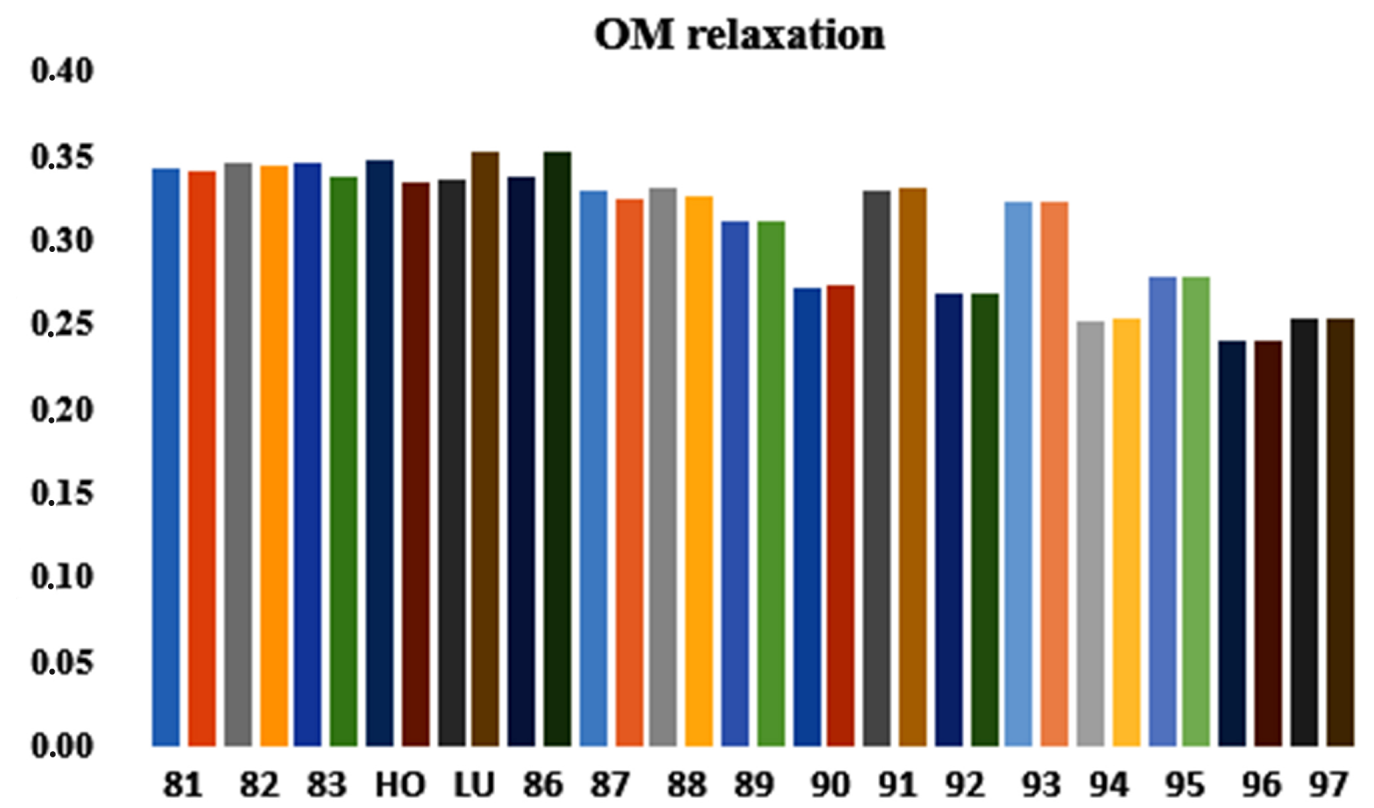

Fig. $6 \mathrm{MO}$ relaxation through the 0.05 - $\mathrm{HOMO}(\# 84) \rightarrow L U M O(\# 85)$ excitation of complex A (ethyl bridge) 


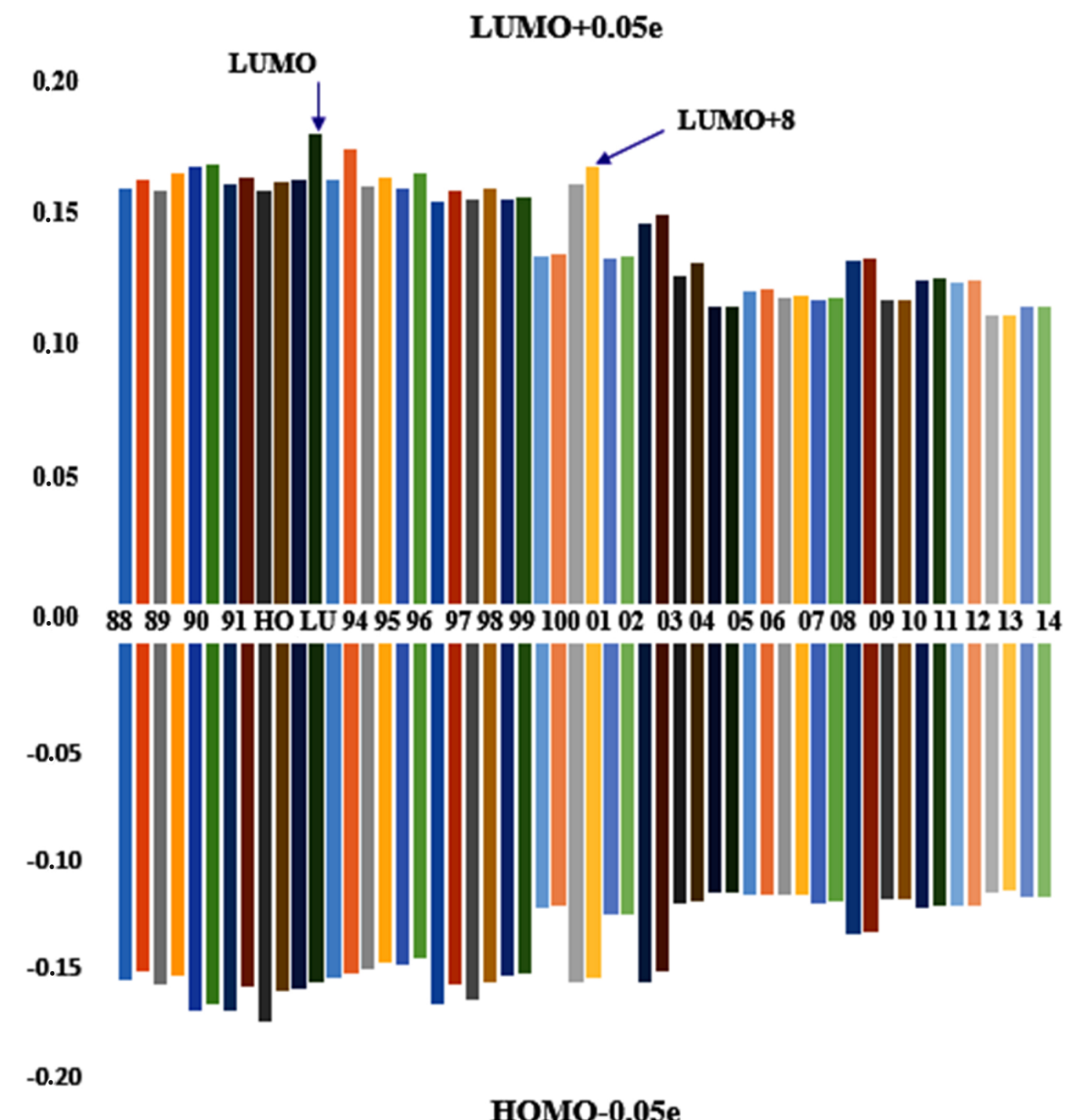

Fig. 7 Relaxation of the $(\alpha, \beta)$ orbitals of the $\boldsymbol{C}$ complex (Phenyl link) when frontier orbitals are altered by 0.05 electron occupancy. (Eigen value shifts in eV). 
LUMO+0.05e

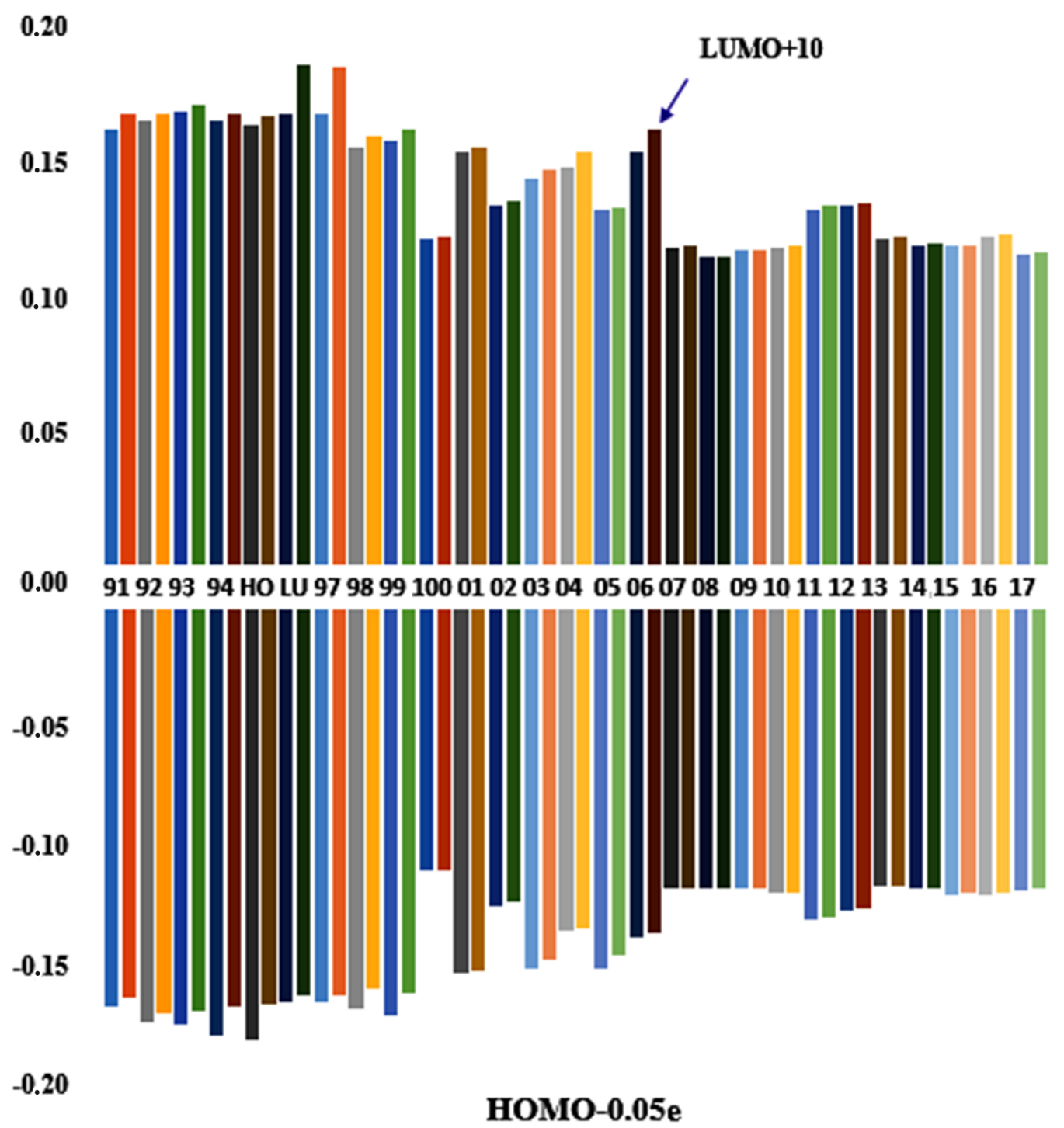

Fig. 8 Relaxation of the $(\alpha, \beta)$ orbitals of the $\boldsymbol{B}$ complex (Cyclohexyl link) when frontier orbitals are altered by 0.05 electron occupancy. (Eigen value shifts in $\mathrm{eV}$ ). 
LUMO + 0.05e

0.20
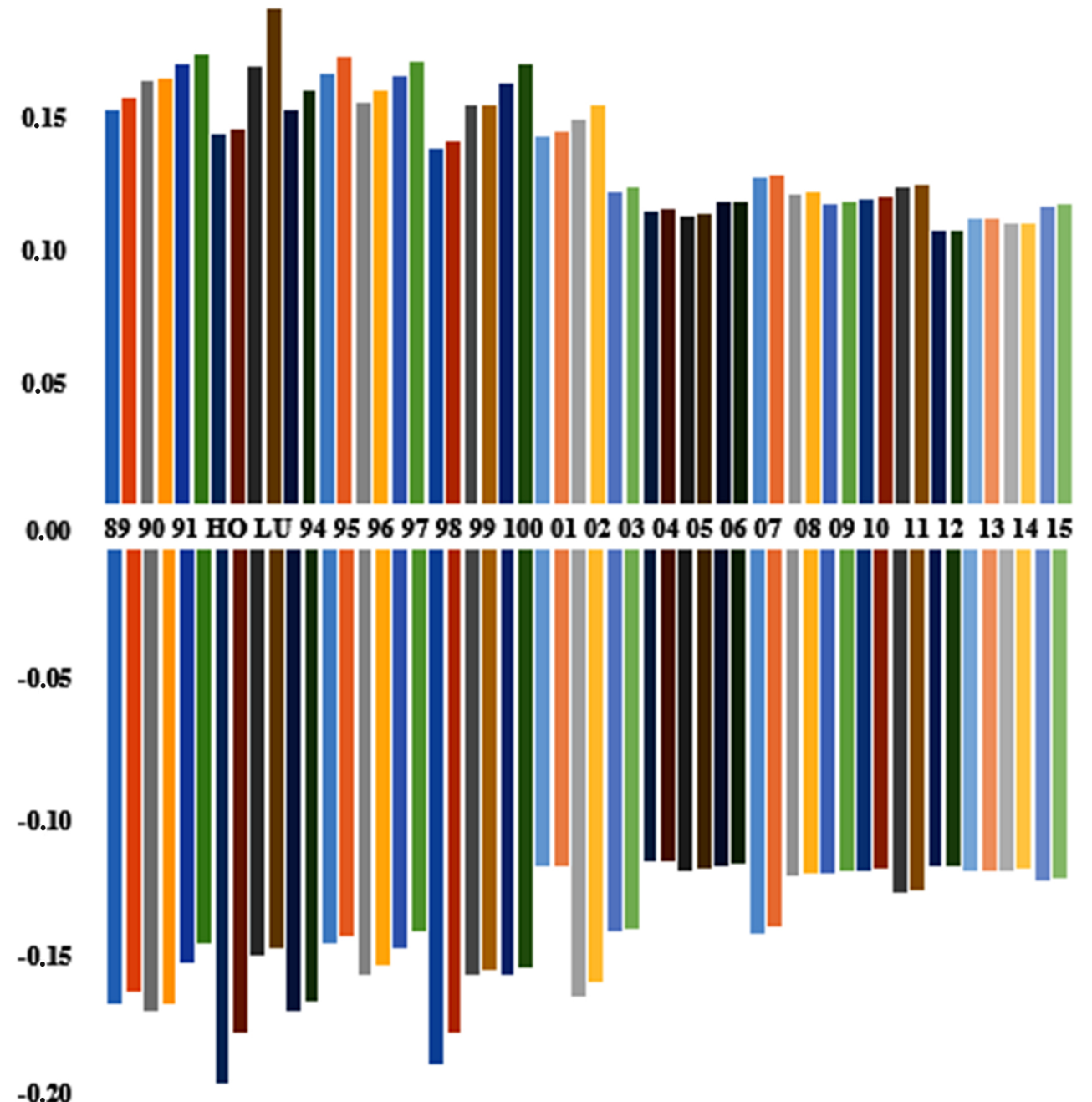

HOMO - 0.05e

Fig. 9 Relaxation of the $(\alpha, \beta)$ orbitals of the $\mathbf{D}$ complex (pyridyl link) when frontier orbitals are altered by 0.05 electron occupancy. (Eigen value shifts in eV). 


\section{Acknowledgments}

The authors gratefully acknowledge the GENCI/CINES for HPC resources/computer time (Project cpt2130) and support from ANR Oxycat-CO2

\section{References:}

1. Lamine W, Boughdiri S, Christ L, Morell C, Chermette H. (2019) J. Comput. Chem. 40:717-725.

2. James E. Huheey, Ellen A. Keiter RLK. (1993) Inorganic Chemistry: Principles of Structure and Reactivity. Harper Collins College Publishers, New York.

3. Morell C, Grand A, Toro-Labbé A. (2005) J. Phys. Chem. A. 109:205-12.

4. Kleij AW, Kuil M, Tooke DM, Lutz M, Spek AL, Reek JNH. (2005) Chem. - A Eur. J. 11:4743-4750.

5. Belmonte MM, Wezenberg SJ, Haak RM, Anselmo D, Escudero-Adán EC, Benet-Buchholz J, Kleij AW. (2010) Dalt. Trans. 39:4541-4550.

6. Matalobos JS, García-Deibe AM, Fondo M, Navarro D, Bermejo MR. (2004) Inorg. Chem. Commun. 7:311-314.

7. Kleij AW, Kuil M, Lutz M, Tooke DM, Spek AL, Kamer PCJ, Van Leeuwen PWNM, Reek JNH. (2006) Inorganica Chim. Acta. 359:1807-1814.

8. Consiglio G, Oliveri I Pietro, Punzo F, Thompson AL, Di Bella S, Failla S. (2015) Dalt. Trans. 44:13040-13048.

9. Mizukami S, Houjou H, Nagawa Y, Kanesato M. (2003) Chem. Commun. (Camb). 91:1148-1149.

10. Zhang G, Li Q, Proni G. (2014) Inorg. Chem. Commun. 40:47-50.

11. Kotova O, Semenov S, Eliseeva S, Troyanov S, Lyssenko K, Kuzmina N. (2009) Eur. J. Inorg. Chem.3467-3474.

12. Consiglio G, Oliveri I Pietro, Failla S, Di Bella S. (2016) Inorg. Chem. 55:10320-10328.

13. Mizukami S, Houjou H, Sugaya K, Koyama E, Tokuhisa H, Sasaki T, Kanesato M. (2005) Chem. Mater. 17:50-56.

14. Lamine W, Boughdiri S, Jeanneau E, Sanglar C, Morell C, Christ L, Chermette H. (2018) ChemPhysChem. 19:2938-2946.

15. Forte G, Oliveri I Pietro, Consiglio G, Failla S, Di Bella S. (2017) Dalt. Trans. 46:4571-4581.

16. Chermette H. (1999) J. Comput. Chem. 20:129-154.

17. Geerlings P, De Proft F, Langenaeker W. (2003) Chem. Rev. 103:1793-873.

18. Perdew JP, Parr RG, Levy M, Balduz JLJ. (1982) Phys. Rev. Lett. 49:1691-1694.

19. Parr RG, Yang W. (1984) J. Am. Chem. Soc. 106:4049-4050.

20. Yang W, Parr RG, Pucci R. (1984) J. Chem. Phys. 81:2862-2863.

21. Tognetti V, Morell C, Ayers PW, Joubert L, Chermette H. (2013) Chem. Chem. Phys. 15:14465.

22. Guégan F, Tognetti V, Joubert L, Chermette H, Luneau D, Morell C. (2016) Phys. Chem. Chem. Phys. 18:982-990.

23. Velde te G, Bickelhaupt FM, Baerends EJ, Fonseca Guerra C, van Gisbergen SJA, Snijders JG, Ziegler T. (2001) J. Comput. Chem. 22:931-967.

24. Baerends EJ, Ziegler T, Atkins AJ, Autschbach J, Bashford D, Baseggio O, Bérces A, Bickelhaupt FM, Bo C, Boerritger PM, Cavallo L, Daul C, Chong DP, Chulhai D V, Deng L, Dickson RM, Dieterich JM, Ellis DE, van Faassen M, Ghysels A, Giammona A, van Gisbergen SJA, Goez A, Götz AW, Gusarov S, Harris FE, van den Hoek P, Hu Z, Jacob CR, Jacobsen H, Jensen L, Joubert L, Kaminski JW, van Kessel G, König C, Kootstra F, Kovalenko A, Krykunov M, van Lenthe E, McCormack DA, Michalak A, Mitoraj M, Morton SM, Neugebauer J, Nicu VP, Noodleman L, Osinga VP, Patchkovskii S, Pavanello M, Peeples CA, Philipsen PHT, Post D, Pye CC, Ramanantoanina H, Ramos P, Ravenek W, Rodríguez JI, Ros P, Rüger R, Schipper PRT, Schlüns D, van Schoot H, Schreckenbach G, Seldenthuis JS, Seth M, Snijders JG, Solà M, M. S, Swart M, Swerhone D, te Velde G, Tognetti V, Vernooijs P, Versluis L, Visscher L, Visser O, Wang F, Wesolowski TA, van Wezenbeek EM, Wiesenekker G, Wolff SK, Woo 
TK, Yakovlev AL. ADF2017, SCM, Theoretical Chemistry, Vrije Universiteit, Amsterdam, The Netherlands, https://www.scm.com.

25. Chermette H. (1982) unpublished.

26. Chermette H. (1992) New J. Chem. 16:1081-1088.

27. Gilbert ATB, Besley NA, Gill PMW. (2008) J. Phys. Chem. A. 112:13164-13171.

28. Barca GMJ, Gilbert ATB, Gill PMW. (2018) J. Chem. Theory Comput. 14:1501-1509. 